\title{
ISLAS DE CALOR Y CONFORT TÉRMICO EN ZARAGOZA DURANTE LA OLA DE CALOR DE JULIO DE 2015
}

\author{
Ernesto TEJEDOR ${ }^{1,2}$, José M. CUADRAT ${ }^{1,2}$, Miguel A. SAZ ${ }^{1,2}$, Roberto \\ SERRANO-NOTIVOLI ${ }^{1,2,3}$, Nieves LÓPEZ ${ }^{4}$, Mariano ALADRÉN ${ }^{4}$ \\ ${ }^{1}$ Departamento de Geografía y Ordenación del Territorio. Universidad de Zaragoza. \\ ${ }^{2}$ Instituto Universitario de Ciencias Ambientales de Aragón. Universidad de Zaragoza. \\ ${ }^{3}$ Estación Experimental de Aula Dei. Consejo Superior de Investigaciones Cientificas. \\ ${ }^{4}$ Agencia de Medio Ambiente y Sostenibilidad. Ayuntamiento de Zaragoza. \\ etejedor@unizar.es
}

\section{RESUMEN}

En este trabajo se analiza la isla de calor y la isla de sequedad del verano del año 2015 a partir de la red de sensores de temperatura y humedad existentes en la ciudad y su entorno inmediato. Asimismo, se evalúa la incidencia de la ciudad sobre las temperaturas y la humedad durante la intensa ola de calor del mes de julio, y se caracteriza el régimen de confort a partir del índice de confort térmico THI, de Thom.

Palabras clave, Isla de Calor, Ola de Calor, Índice termohigrométrico de confort (THI), Clima Urbano, Zaragoza.

\begin{abstract}
This paper analyzes both the heat island and the island of dryness of the summer of 2015 from the network of temperature and humidity sensors existing in the city and its surroundings. Furthermore, we evaluate the incidence of the city within the temperatures and humidity during the intense July heat wave. Finally, we characterized the thermal comfort index from the thermal comfort index THI, Thom.
\end{abstract}

Key words, Urban Heat Island, Heatwave, Thermohygrometric index (THI), Urban Climate, Zaragoza.

\section{INTRODUCCIÓN Y ANTECEDENTES}

Los procesos de urbanización generan cambios sustanciales en la cubierta vegetal y los usos del suelo, así como interferencias en los flujos de energía entre la atmósfera y las distintas superficies, alterando las características del clima local. Los efectos más conocidos son las denominadas isla de calor urbana (UHI, Urban Heat Island) (Oke, 1995; Arnfield, 2003) e isla de sequedad (UDI, Urban Dry Island) (Lee, 1991; Jáuregui y Tejeda, 1997; Robaa, 2013, Cuadrat et al, 2015), que se manifiestan en una clara anomalía termohigrométrica entre el interior de las ciudades y su periferia. Su intensidad y características está relacionada con el tamaño y población de la ciudad (Hogan y Ferrick, 1988), y por lo general, es mayor en verano que en invierno (Morris et al., 2001). Son muy evidentes durante la noche (Jauregui, 1997), llegando a desaparecer durante el día (Steinecke, 1999). 
Desde el punto de vista del confort térmico la presencia de islas de calor e islas de sequedad en las ciudades puede tener efectos importantes sobre la calidad de vida de sus habitantes e incluso repercusiones sobre la salud humana, como estrés térmico o mayor exposición a los contaminantes, siendo conocido el incremento registrado en la mortalidad cuando se superan los $36^{\circ} \mathrm{C}$ (Díaz et al, 2015).

La configuración e intensidad de UHIs y UDIs están determinadas por factores geográficos y estructurales. Por ejemplo, los parques aparecen como zonas relativamente frías y con mayor humedad relativa en comparación con las áreas construidas de su entorno; de hecho, la cubierta vegetal afecta de modo evidente la distribución de las temperaturas y la humedad e interviene directamente en las condiciones de confort. E igualmente relevante es la acción del relieve, la geometría urbana o el tamaño y población de la ciudad. Sin embargo, sus características no son estables, y la variabilidad espacial y temporal puede ser muy acusada, estando relacionados estos cambios con la dinámica atmosférica regional y local.

Desde esta perspectiva, el conocimiento y monitorización del clima urbano tiene un enorme interés, tanto por su valor aplicado para la calidad ambiental de las ciudades, como por ser ejemplo de modificación climática no intencionada.

La ciudad de Zaragoza, situada en el cuadrante nororiental de España en la confluencia de los ríos Gállego y Huerva, tiene una topografía sin grandes contrastes, una textura urbana variada y un clima mediterráneo con una marcada influencia continental (Cuadrat et al, 2007), con escasas precipitaciones $(326 \mathrm{~mm}$ ) y un acusado contraste entre el frío invierno $\left(6,2^{\circ} \mathrm{C}\right.$ es la temperatura media de enero) en el que son frecuentes las situaciones de inversión térmica que favorecen la presencia de UHIs y UDIs, y un verano cálido y continuado $\left(24,3{ }^{\circ} \mathrm{C}\right.$ de media en julio) en el que no es inhabitual la presencia de advecciones meridianas de aire cálido, que combinadas con situaciones posteriores de estabilidad atmosférica pueden generar olas de calor persistente durante varios días.

En la primera década de la presente centuria se realizaron estudios de las islas de calor y sequedad de Zaragoza en los que se caracterizó espacialmente su configuración, se cuantificó la diferencia entre las áreas más cálidas de la ciudad y la periferia en determinadas situaciones atmosféricas (próxima a los $6^{\circ} \mathrm{C}$ en algunos momentos), se comprobó la relación entre la localización de las islas de calor y sequedad con el flujo de vientos en superficie y se analizó su variabilidad espacial (Cuadrat, 2004; Cuadrat et al, 2003, 2005, 2015; López-Martín, 2011; Saz et al, 2003; Vicente et al, 2003, 2005).

Las mediciones se hacían por medio de transectos urbanos, normalmente unas tres horas después de la puesta de sol y con registro en 216 puntos, que ofrecían una imagen termohigrométrica fija de la ciudad en ese momento. Esta metodología de toma de datos no permite una monitorización continua de la evolución de UHI y UDI y por tanto su puesta en relación con otros factores, como la evolución de los contaminantes, la intensidad del tráfico, picos de mortalidad o la variación de ambos fenómenos en relación con los cambios en las situaciones atmosféricas.

Además, tras la celebración en Zaragoza de la Exposición Internacional de 2008 la estructura urbana de la ciudad ha cambiado significativamente, abandonando en parte la trama compacta que la caracterizaba, aumentando los espacios verdes en el interior y la periferia y cerrándose las circunvalaciones viales, lo que junto a la 
reciente instalación del tranvía han reducido la circulación de vehículos en el anillo central de la ciudad.

Para analizar las consecuencias de estos cambios, en el año 2015, en colaboración con la Agencia de Medio Ambiente y Sostenibilidad del Ayuntamiento de Zaragoza, se instalaron sensores de temperatura y humedad en diferentes puntos de la ciudad y su entorno que permiten profundizar en el conocimiento de las condiciones climáticas urbanas y sus patrones actuales. En este trabajo se presentan los primeros avances del análisis más preciso de la isla de calor y la isla de sequedad estivales, sus modificaciones durante la ola de calor de julio de 2015 y su impacto sobre el grado de confort de la población durante este periodo cálido extremo, en el que el observatorio de Zaragoza registró la temperatura máxima absoluta desde que existen datos instrumentales.

\section{DATOS Y MÉTODOS}

\subsection{Base de datos}

Los datos utilizados en este trabajo se han obtenido de la red de sensores termohigrométricos instalados en el año 2015 en el interior de la ciudad y su entorno. Los aparatos son del tipo HOBOpro v2 (fig 1A), con sensor de temperatura y humedad, y un data-logger para el almacenamiento de la información. Su rango de funcionamiento en las temperaturas es de los $-40^{\circ} \mathrm{C}$ a los $70{ }^{\circ} \mathrm{C}$, con una precisión de $0,21^{\circ} \mathrm{C}$ y una resolución de 0,02 grados. Para la humedad relativa del aire el rango operacional del sensor es de $0-100 \%$ y su precisión del $2,5 \%$. Para protegerlos de la radiación solar directa y del efecto de la lluvia, los sensores se montaron dentro de un soporte tipo M-RSA (fig 1B).

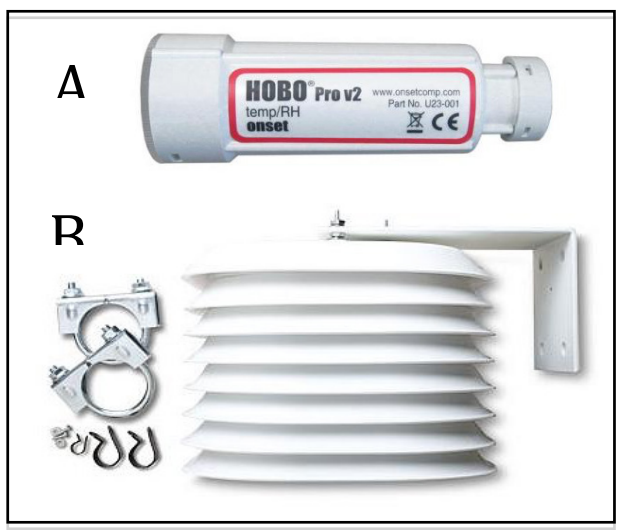

Fig. 1. Sensores tipo HOBO utilizados y elemento de protección frente a la radiación solar directa.

En la actualidad (Junio de 2016) la red está formada por 21 sensores, aunque para la realización de este trabajo se han utilizado 15 sensores (fig 2), ubicados en lugares representativos de distintos ambientes dentro de la ciudad y su periferia inmediata, y convenientemente alejados de posibles fuentes de calor o de superficies que pudieran irradiar calor hacia el sensor, en especial durante la noche. 


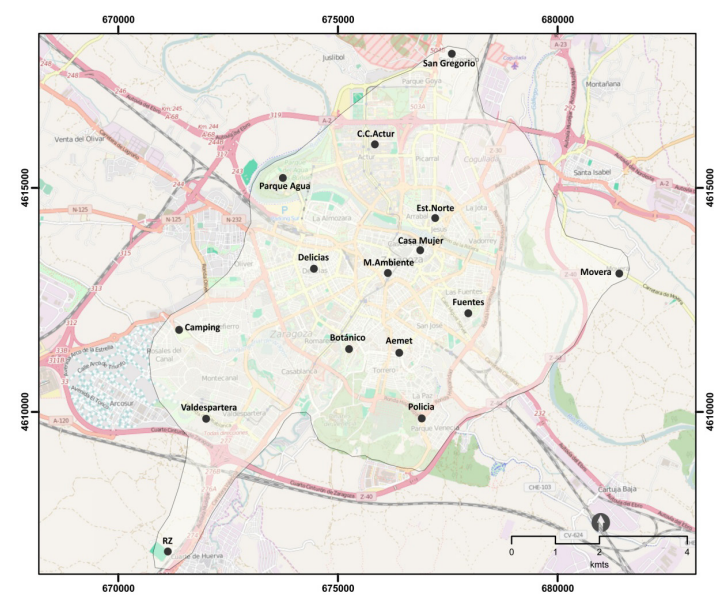

Fig. 2. Mapa de ubicación de los sensores.

\subsection{Análisis preliminar y depuración de los datos}

Una vez descargados los datos asistidos por el software Onset HOBOware vs 3.7, estos fueron sometidos a un riguroso control de calidad, desarrollado en lenguaje $\mathrm{R}$ en el que se ha evaluado la presencia de lagunas de información, datos aberrantes o inhomogeneidades. Una vez depurados los registros, se analizaron utilizando el package stats de R-Studio para calcular valores estadístico-descriptivos de las series.

\subsection{Cálculo del índice de confort}

Para cuantificar las sensaciones de confort y establecer escalas que permitan determinar las respuestas de la población ante unas condiciones climáticas específicas se ha utilizado el índice THI (Thom, 1959). Este índice indica la temperatura que con aire en calma provocaría en una persona en reposo la misma sensación que la temperatura y humedad reales. Se ha utilizado en numerosos trabajos para evaluar las diferencias entre espacios rurales y urbanos (Jauregui y Tejada, 1997; Fernández, 2002) y es sencillo de calcular a partir de la ecuación:

$$
\text { THI }=t-(0.55-0.0055 H) *(t-14.5)
$$

Siendo $t$ la temperatura del aire, en ${ }^{\circ} \mathrm{C}$ y $H$ la humedad relativa, en $\%$

\subsection{Cartografía}

Para la representación cartográfica de los datos se ha utilizado ArcGIS 10.2. En el proceso de interpolación se aplicaron cuatro métodos, inverso a la distancia, funciones radiales, kriging simple y kriging ordinario. El proceso se ha desarrollado utilizando el asistente geoestadístico de ArcGIS 10.2. La validación y selección de la mejor cartografía viene de la mano del cálculo del RMSE mediante un proceso de validación cruzada. La cartografía finalmente elegida como más representativa para cada variable nos la dará el valor más bajo obtenido para el RMSE. 


\section{RESULTADOS Y DISCUSIÓN}

\subsection{Configuración espacial de la UHI y la UDI}

La isla de calor es un fenómeno esencialmente nocturno, cuando el calor almacenado por el asfalto y edificios en el interior de la ciudad es remitido a la atmósfera limitando su enfriamiento. Por el contrario, durante el día las diferencias campo-ciudad se reducen y la isla puede llegar a desaparecer e incluso provocar una verdadera isla de frescor. Un ritmo muy similar es el que sigue la humedad relativa del aire y la isla de sequedad. Aunque su presencia se observa a lo largo de todo el año, para su análisis se ha considerado exclusivamente el periodo estival, desde junio a septiembre, por su mayor impacto sobre el estrés térmico y para conocer su intensidad durante los días de olas de calor.

El ciclo diario muestra el momento máximo de las islas de calor y sequedad entre las 3 y 4 horas de la madrugada (hora local) y disminuye progresivamente hasta media tarde. En el caso de las temperaturas, a las 7:00 h la UHI se observa todavía con claridad en torno a dos puntos centrales, el barrio de Delicias y el eje que va del entorno del Paseo de Independencia-Plaza Aragón hasta la Plaza Santa Marta. Son zonas de edificación compacta, que ya en el estudio de 2002 mostraban un ambiente más cálido. Las áreas más frescas se sitúan en la periferia de la ciudad, como el Barrio rural de San Gregorio y Movera, así como en las zonas urbanas ajardinadas del Parque Grande y Parque del Agua, donde la vegetación y la mayor superficie arbolada amortiguan el efecto de las temperaturas máximas (fig 3).

En el caso de la UDI los valores con menor humedad se encuentran en el centro de la ciudad, siendo el entorno de los observatorios de Delicias, Casa de la Mujer y Medio Ambiente los que presentan una humedad más baja respecto a la media. Asimismo, los valores más altos los encontramos al norte del río Ebro, destacando el Parque del Agua con un 6\% de media por encima del promedio.
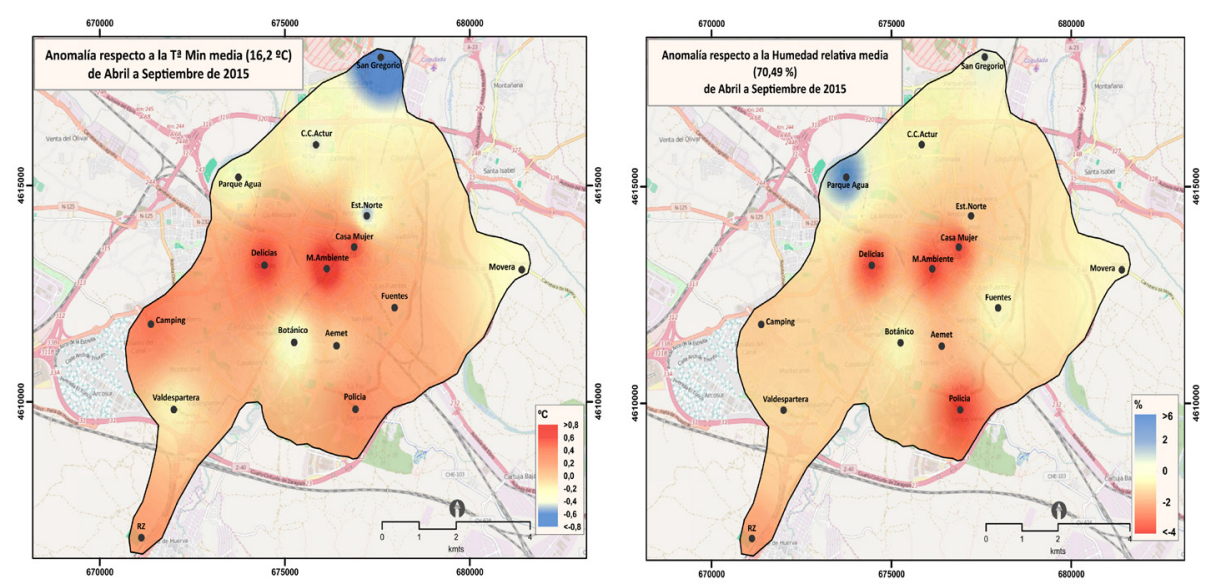

Fig. 3. Isla de Calor de Zaragoza (valores promedio de Temperatura y Humedad relativa a las 7:00h entre junio y septiembre de 2015) 


\subsection{La ola de calor de julio de 2015}

En el ámbito regional del Valle del Ebro en el que se ubica la ciudad de Zaragoza, en verano, y principalmente en los meses de julio y agosto, es común que las temperaturas diarias igualen o superen $\operatorname{los} 30^{\circ} \mathrm{C}$. Pero además, en situaciones atmosféricas de tipo anticiclónico o con régimen de vientos del Sur, a veces de origen sahariano, el aire cálido se estanca en el fondo de la cubeta del Valle, y en ausencia de movimientos horizontales en los niveles bajos de la atmósfera, aquéllas se elevan de modo progresivo hasta alcanzar registros de 38 a $40{ }^{\circ} \mathrm{C}$ en las máximas y de 20 a $22^{\circ} \mathrm{C}$ en las mínimas, que pueden prolongarse durante varios días.

Uno de estos periodos cálidos excepcionales se produjo a finales del mes de junio y primeros de julio de 2015. Durante varios días en todos los puntos de observación instalados en la ciudad se superaron los $35^{\circ} \mathrm{C}$, y las condiciones de confort según el índice THI alcanzaron niveles considerados como "muy cálidos" e incluso "bochornosos". La ola de calor llegó a su máximo el día 7 de julio. En esta jornada, en el observatorio meteorológico de AEMET, situado en el Aeropuerto, se registró la temperatura máxima histórica desde que existen observaciones instrumentales: $44,5^{\circ} \mathrm{C}$; y en prácticamente todos los sensores las temperaturas se aproximaron o superaron los $44^{\circ} \mathrm{C}$, entre las 16 y las 18 horas (fig. 4).

La cartografía de la temperatura y la humedad en diferentes momentos del día muestra de nuevo la presencia de las islas de calor y sequedad, pero sobre todo indica que durante las olas de calor el calentamiento urbano es aún más evidente. En todo momento el interior urbano es más cálido y se mantiene más seco, mientras la periferia se conserva más fresca y húmeda, en particular el espacio arbolado y próximo al río del Parque del Agua, cuya temperatura siempre fue entre $1-2^{\circ} \mathrm{C}$ más baja (fig. 5).

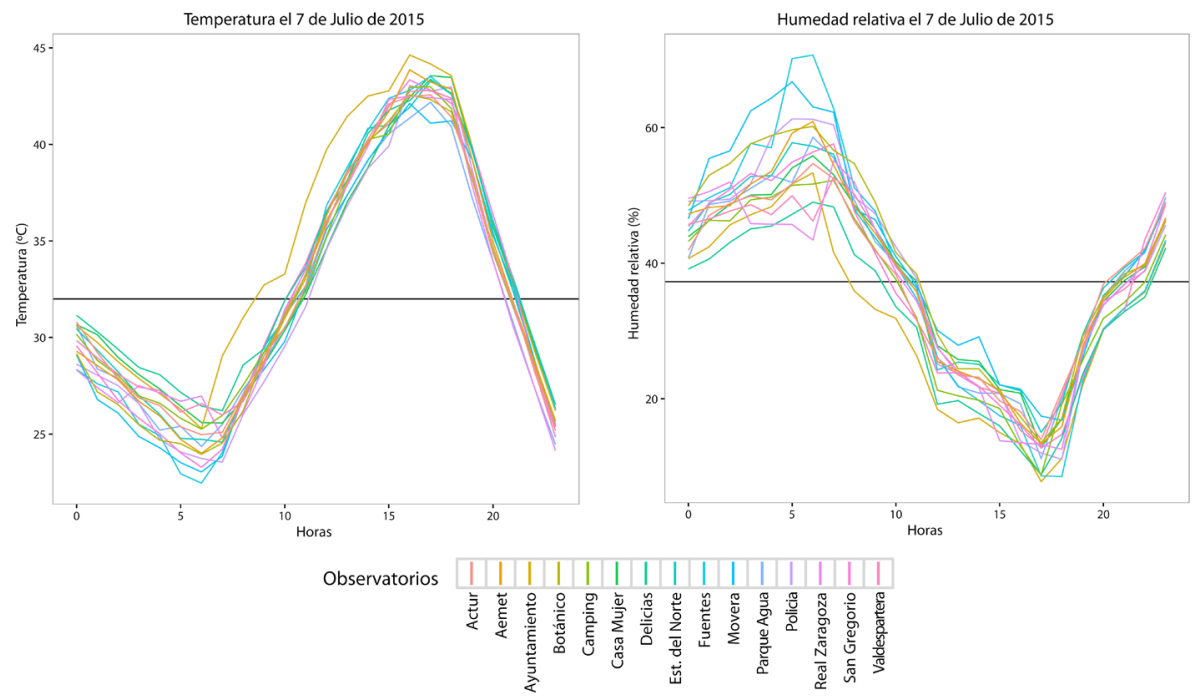

Fig. 4. Evolución de las temperaturas horarias en los distintos observatorios el día 7 de julio 2015. 

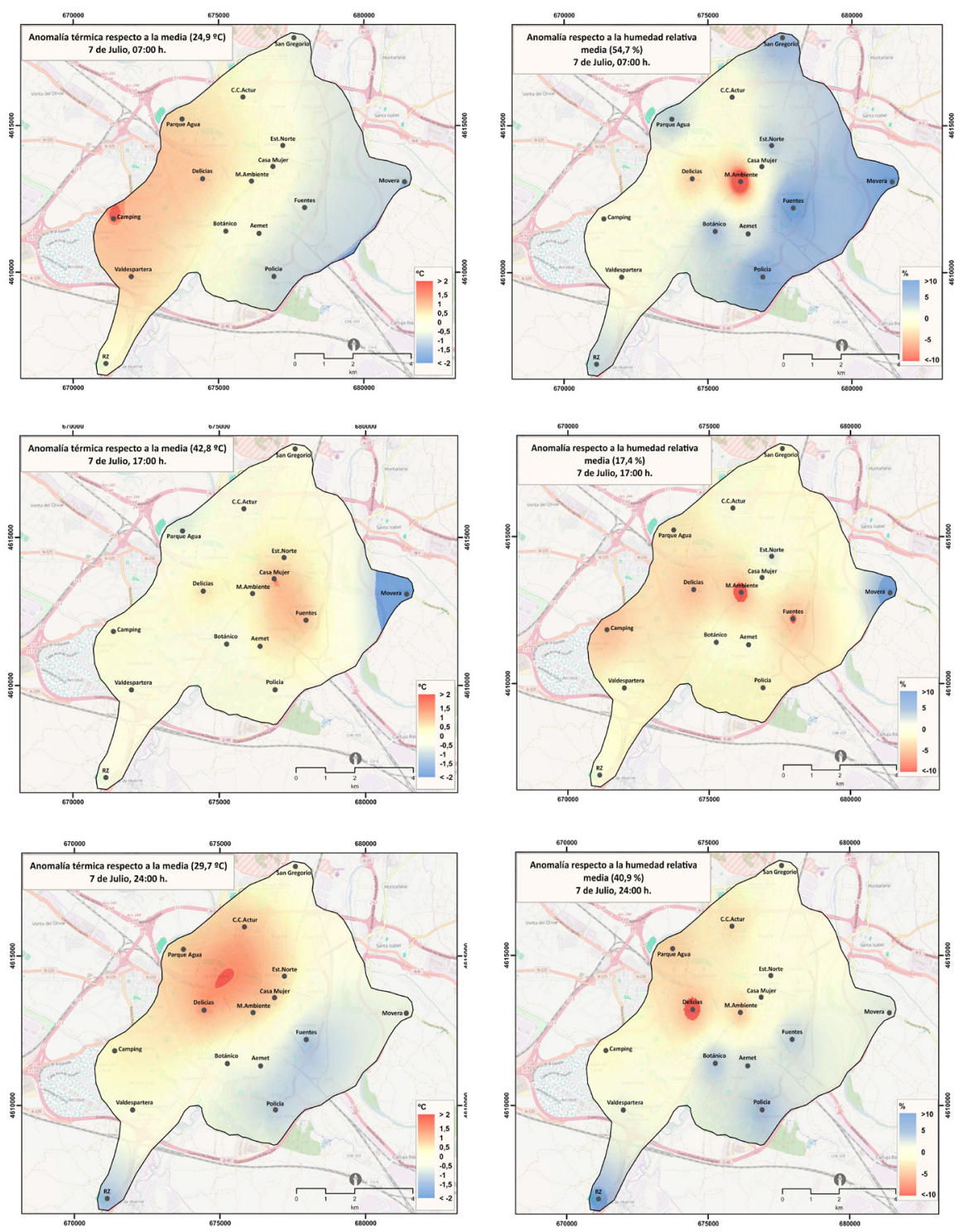

Fig. 5. UHI y UDI en Zaragoza a las 7h, 17 h y 24 h del dia 7 de julio 2015

A medianoche toda la ciudad rozaba e incluso rebasaba los $30{ }^{\circ} \mathrm{C}$; en consecuencia, el grado de confort era globalmente de cálido, y en varias zonas llegaba a muy cálido y también bochornoso (fig. 6).

Para evaluar la incidencia de la ciudad durante la ola de calor hemos comparado la intensidad de la isla de calor en el conjunto de los días estivales y en aquellos días en los que la temperatura máxima supera los $36,5^{\circ} \mathrm{C}$. Para ello se ha representado el 
ciclo diario de la isla de calor propia de los días de verano (obtenida comparando los valores térmicos horarios del conjunto de los sensores ubicados en el interior de la ciudad con los de la periferia) y el correspondiente sólo a los días en los que la temperatura en el exterior de la urbe ha alcanzado $\operatorname{los} 36,5^{\circ} \mathrm{C}$ (umbral a partir del cual entra en vigor un sistema de alerta por ola de calor).

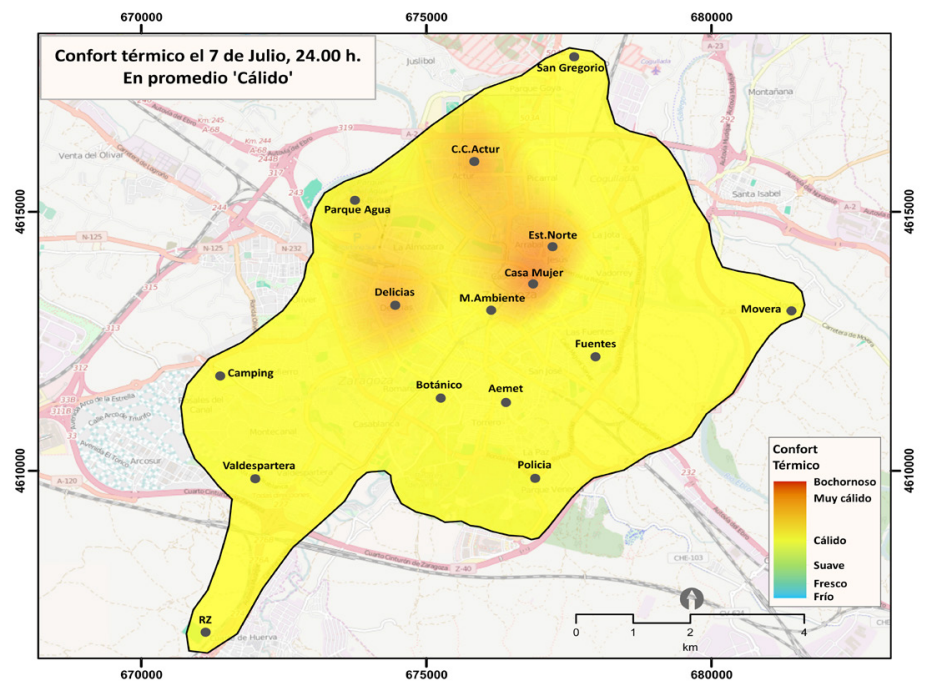

Fig. 6. Índice de confort THI de Thom, a las 24 h del dia 7 de julio 2015

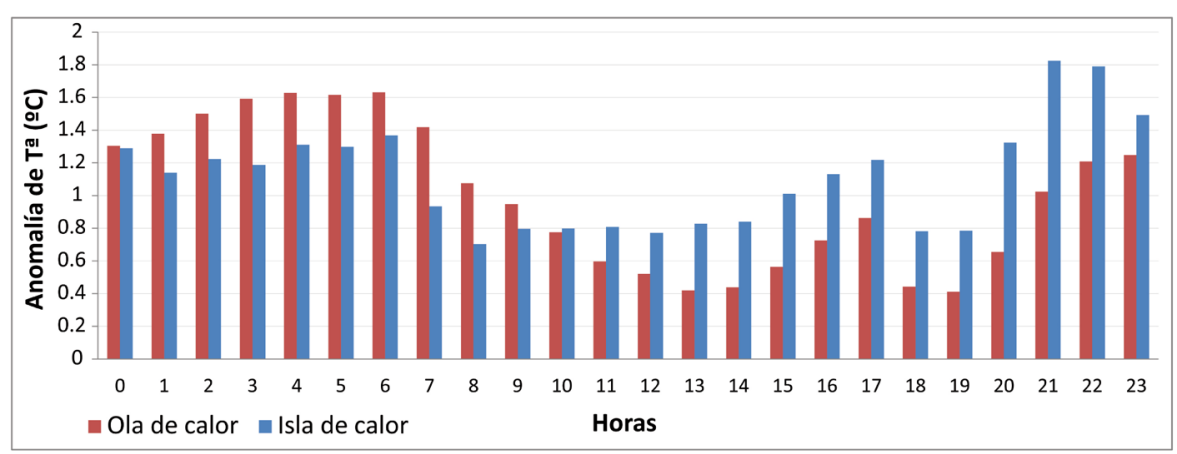

Fig. 7. Ciclo diario de la isla de calor media y durante los días cálidos

Los resultados (fig. 7) muestran que la intensidad de la isla de calor aumenta sensiblemente durante los días cálidos, particularmente en el caso de las temperaturas mínimas, y algo menos en las máximas. El ciclo diario muestra la existencia de una débil isla de calor a mediodía, aumenta su desarrollo a partir de las 17 h y se mantiene con valores altos por la noche hasta las $6 \mathrm{~h}$ de la madrugada, momento en el cual las zonas rurales comienzan a recibir radiación solar y el ascenso de la temperatura en ellas es más rápido que en la ciudad. Los días de ola de calor la evolución de la isla 
es bastante similar, pero su intensidad es mayor durante las horas nocturnas: prácticamente durante toda la noche y hasta bien entrada la mañana la isla de calor de los días cálidos es muy superior a la isla promedio.

\section{CONCLUSIONES}

El rasgo más característico del verano en Zaragoza es el calor, de tal modo que las temperaturas máximas alcanzan los $30^{\circ} \mathrm{C}$ buena parte de los meses estivales y existe un número de días relativamente alto en los que se superan $\operatorname{los} 35^{\circ} \mathrm{C}$, lo cual tiene una incidencia directa sobre el confort de la población.

Pero además, estas condiciones se agravan en el interior de la ciudad, donde las temperaturas son más altas que las de su entorno rural inmediato por el efecto conocido como isla de calor.

La base de datos disponible permite comprobar como las zonas de mayor densidad de edificación registran temperaturas por encima de la media general casi todo el día. Así ocurre en el centro de la ciudad, el barrio de las Delicias y el entorno Plaza Aragón hasta la Plaza de Santa Marta. En sentido opuesto, las zonas ajardinadas, las de densidad urbana baja y los barrios rurales de la periferia presentan valores inferiores a la media general. El dibujo es muy similar al que ofrece el patrón de distribución de la humedad relativa, por su fuerte correspondencia con las temperaturas, con la presencia de una clara isla de sequedad en el centro urbano que contrasta con las condiciones más húmedas del entorno rural.

El fenómeno se produce fundamentalmente de noche, mientras que durante el día las diferencias campo-ciudad se ven bastante atenuadas. Durante los días de calor extremo, en el transcurso de olas de calor, la UHI nocturna incrementa su intensidad

Estas condiciones tienen un notable impacto sobre el confort térmico, de modo que en los meses estivales, y en particular en las olas de calor, el interior urbano presenta condiciones calificadas como cálidas, y que llegan a ser muy cálidas o bochornosas en las zonas más significativas de localización de la isla de calor.

En resumen, en los periodos cálidos la ciudad de Zaragoza agudiza de forma considerable el calor y la sequedad en relación a las zonas no urbanas. En sentido opuesto, las superficies ajardinadas y con formaciones arbóreas de cierta entidad, como el Parque del Agua o Parque Grande José Antonio Labordeta, actúan de forma significativa como moderadores del estrés térmico.

\section{AGRADECIMIENTOS}

Este trabajo ha sido posible gracias a los proyectos 909.338/2014 Patrones temporales y espaciales de la temperatura urbana de Zaragoza, y 943.674/2013 Clima y masas forestales del término municipal de Zaragoza en el contexto del cambio global, financiados por la Agencia de Medio Ambiente y Sostenibilidad del Ayuntamiento de Zaragoza. Ha contado también con la ayuda del Gobierno de Aragón y Fondo Social Europeo al grupo de investigación "Clima, agua y cambio global” de la Universidad de Zaragoza. 


\section{REFERENCIAS}

Arnfield, AJ. (2003). Two decades of urban climate research, a review of turbulence, exchanges of energy and water, and the urban heat island. International Journal of Climatology 23, 1-26.

Cuadrat, J.M., Saz, M.A. y Vicente-Serrano, S. (2003). Surface wind direction influence on spatial patterns of urban heat island in Zaragoza (Spain). Geophysical Research Abstracts, 5, 02592. European Geophysical Society

Cuadrat, J.M. (2004). Patrones temporales de la isla de calor urbana de Zaragoza. En, Faus, M.C. (Coord.). Aportaciones geográficas en homenaje al profesor Higueras. Universidad de Zaragoza. Zaragoza, 63-70.

Cuadrat, J.M.; Vicente, S. y Saz, M.A. (2005). Los efectos de la urbanización en el clima de Zaragoza (España), la isla de calor y sus factores condicionantes. Boletín de la Asociación de Geógrafos Españoles, 40, 311-327

Cuadrat, J.M., Saz, M.A., Vicente, S.M. (2007). Atlas Climático de Aragón. 222 pp.

Cuadrat, J.M., Vicente-Serrano, S., Saz, M.A. (2015). Influence of different factors on relative air humidity in Zaragoza, Spain. Frontiers in Earth Science, 3 (10).

Díaz, J., Carmona, R., Mirón, I.J., Ortiz, C., Linares, C. (2015). Comparison of the effects of extreme temperatures on daily mortality in Madrid (Spain), by age group: The need for a cold wave prevention plan. Environmental Research. 143, 186-191.

Fernández, F. (2002). El clima urbano de Madrid y su influencia sobre el confort térmico. Boletín de la Real Sociedad Geográfica, CXXXVIII, 169-185.

Hogan, A.W., Ferrick, M.G. (1988). Observations in nonurban heat islands. Journal of Applied Meteorology 37, 232-236.

Jauregui, E. (1997). Heat island development in Mexico City. Atmospheric Environment 31, 3821-3831

Jauregui, E. Tejeda, A. (1997). Urban-rural humidity contrast in Mexico City.

Lee, D. (1991). Urban-rural humidity differences in London. International Journal of Climatology, 11, pp. 577-582

López Martín, F. (2011). Clima urbano y ciudad. El caso de Zaragoza. Colegio de Geógrafos. Zaragoza, $118 \mathrm{pp}$

Morris, C.J., Simmonds, I., Plummer, N. (2001). Quantification of the influences of wind and cloud on the nocturnal urban heat island of a large city. Journal of Applied Meteorology 40, 169-182

Oke, T.R. (1995). The heat island of the urban boundary layer, characteristics, causes and effects. In Wind Climate in Cities. Cermak, J.E. et al. (eds.). KluwerAcademic Publ. Norwell Ma.

Robaa, S.M. (2013). Some aspects of the urban climates of Greater Cairo Region, Egypt. International Journal of Climatology, 33, 3206-3216.

Saz, M.A., Vicente, S. y Cuadrat, J.M. (2003). Spatial patterns estimation of urban heat island of Zaragoza (Spain) using GIS. Fifth International Conference on Urban Climate. Lodz (Poland), pp 409-412. 
Steinecke, K. (1999). Urban climatological studies in the Reykjavík subartic environment, Iceland. Atmospheric Environment 33, 4157-4162

Thom, E. C. (1959). The discomfort index, Weatherwise 12(2), 57-60.

Vicente, S., Cuadrat, J.M. y Saz, M.A. (2005). Spatial patterns of urban heat island in Zaragoza (Spain). Climate Research 30, 61-69.

Vicente, S., Cuadrat, J.M. y Saz, M.A. 2003. Topography and vegetation cover influence on urban heat island of Zaragoza (Spain). Fifth International Conference on Urban Climate. Lodz (Poland). 